\title{
One-dimensional Tamm plasmons: Spatial confinement, propagation, and polarization properties
}

\author{
I. Yu. Chestnov, ${ }^{1}$ E. S. Sedov, ${ }^{1,2}$ S. V. Kutrovskaya, ${ }^{1,3}$ A. O. Kucherik, ${ }^{1}$ S. M. Arakelian, ${ }^{1}$ and A. V. Kavokin ${ }^{2,3,4,5}$ \\ ${ }^{1}$ Department of Physics and Applied Mathematics, Vladimir State University named after A. G. and N. G. Stoletovs, \\ 87 Gorkii Street, 600000 Vladimir, Russia \\ ${ }^{2}$ Physics and Astronomy School, University of Southampton, Highfield, Southampton SO17 1BJ, United Kingdom \\ ${ }^{3}$ Russian Quantum Center, 100 Novaya Street, 143025 Skolkovo, Moscow Region, Russia \\ ${ }^{4}$ CNR-SPIN, Viale del Politecnico 1, I-00133 Rome, Italy \\ ${ }^{5}$ Spin Optics Laboratory, St. Petersburg State University, St. Petersburg 198504, Russia
}

(Received 25 September 2017; revised manuscript received 1 December 2017; published 26 December 2017)

\begin{abstract}
Tamm plasmons are confined optical states at the interface of a metal and a dielectric Bragg mirror. Unlike conventional surface plasmons, Tamm plasmons may be directly excited by an external light source in both TE and TM polarizations. Here we consider the one-dimensional propagation of Tamm plasmons under long and narrow metallic stripes deposited on top of a semiconductor Bragg mirror. The spatial confinement of the field imposed by the stripe and its impact on the structure and energy of Tamm modes are investigated. We show that the Tamm modes are coupled to surface plasmons arising at the stripe edges. These plasmons form an interference pattern close to the bottom surface of the stripe that involves modification of both the energy and loss rate for the Tamm mode. This phenomenon is pronounced only in the case of TE polarization of the Tamm mode. These findings pave the way to application of laterally confined Tamm plasmons in optical integrated circuits as well as to engineering potential traps for both Tamm modes and hybrid modes of Tamm plasmons and exciton polaritons with meV depth.
\end{abstract}

\section{INTRODUCTION}

The control of optical signals and their manipulation on a micrometer scale are among the important tasks of nanophotonics, especially in the context of the development of all-optical computing technologies. Although dielectric fibers are irreplaceable for long-range optical communications, they are hardly usable for the realization of compact signalprocessing circuits. Thus engineering small and efficient optical devices on a chip is a primary goal of modern photonics. The main hopes in this field are now linked to plasmonic waveguide structures [1] in which light is tightly concentrated near the metal surface due to its coupling to collective excitations of the electron plasma. Because of their capacity for light localization on the subwavelength scale surface plasmons (SPs) are promising candidates for creation of extremely compact optical circuits. However, plasmonic modes are subject to high Ohmic losses which restrict their propagation length to a few tens of wavelength. Furthermore, due to the inherent evanescent nature of plasmonic modes a grating or a prism is necessary for their excitation. Both these factors strongly limit practical applications of plasmonic waveguides.

A somewhat different approach to the localization of light is based on using interfaces between dielectric or semiconductor Bragg mirror (BM) surfaces and metallic films. In this case, light is confined by the dielectric and metallic mirrors, and it decays exponentially on both sides of the metal-dielectric interface. Such localized modes of the electromagnetic field are referred to as Tamm plasmons [2] (TPs) by analogy with localized electronic states at the surface of crystals predicted by Tamm [3]. In contrast to conventional SPs, TPs can be directly optically excited at any angle of incidence in both TM and TE polarizations as their in-plane dispersion lies completely within the light cone. But the main advantage of
TPs over surface plasmons is connected to the lower level of losses which originates from the fact that the field concentrates mostly within the nonabsorbing dielectric BM. For this reason, the linewidth of TPs is typically an order of magnitude lower than that of a SP.

Since the first prediction [2] and experimental observation [4] of TPs, a number of applications for this novel type of surface electromagnetic waves have been proposed. Most of them are based on a TP coupling with other excitations, including a SP [5-7], Bragg cavity mode [8,9], exciton confined either in a quantum well [10] embedded inside the $\mathrm{BM}$ or in a monolayer of the transition-metal dichalcogenide $[11,12]$, and also point emitters like quantum dots [13], organic molecules [14], etc. The strong field enhancement in the vicinity of a surface allows for attaining the strong-coupling regime $[10,11]$, while a comparatively small mode volume of TPs localized beneath the small metal disk allows using the resonant TP structures to control the spontaneous emission of a semiconductor quantum dot through the Purcell effect [13]. The latter phenomenon can also be used for creation of single-photon sources [15]. In the case of a strong coupling with exciton-polariton modes of semiconductor microcavities TPs cause formation of hybrid modes of Tamm plasmons and exciton polaritons which acquire energy shifts with amplitudes proportional to the strength of the coupling [8,9]. For the lowest mode supporting the polariton condensation the magnitude of this shift may reach tens of meV. It paves the way for engineering deep lateral trapping potentials for exciton polaritons by means of the precise deposition of a metal on the surface of a Bragg mirror.

Until now, with the exception of some specific configurations $[16,17]$, TPs were mostly considered in planar twodimensional structures, where a continuous metallic layer is deposited on top of a BM. There are few works aimed at the creation of new compact laser sources which exploit spatial 
(a)

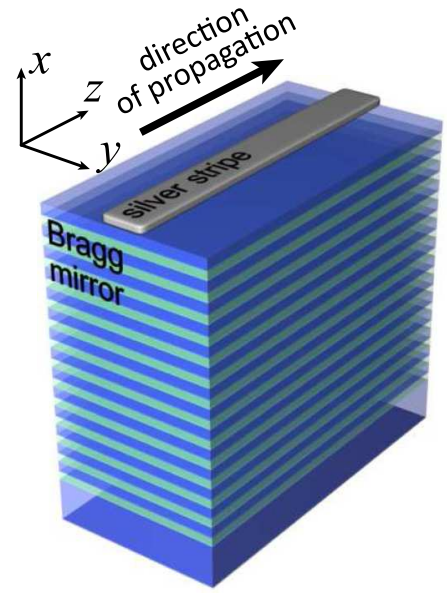

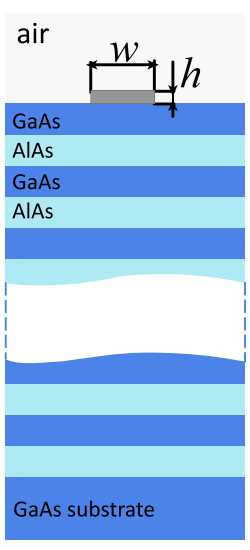

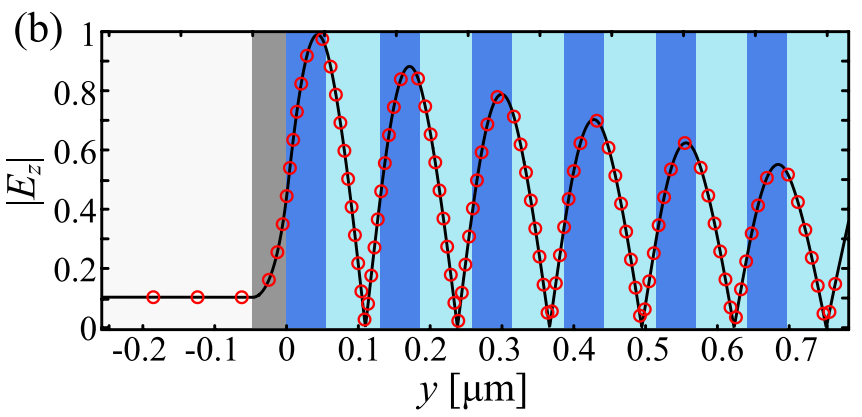

(c)
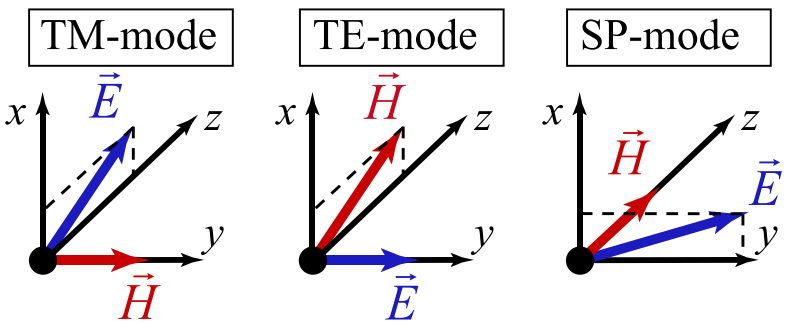

FIG. 1. (a) Sketch of the Bragg mirror with the metal stripe deposited on the top surface. (b) Field distribution of the TM Tamm plasmon mode for the case of a continuous-metal (infinite in the $y-z$ plane) silver film with a thickness $h=50 \mathrm{~nm}$. Circles correspond to the numerical simulations with COMSOL, while the line is obtained using the transfer-matrix method. (c) Orientation of electric $\vec{E}$ and magnetic $\vec{H}$ vectors for TE and TM waveguide Tamm modes propagating along the $z$ axis. The right panel shows the same for the SP mode propagating along the $y$ axis with the wave vector $k_{\mathrm{sp}} \gg k_{z}$.

confinement of TPs beneath a small metallic island $[13,18,19]$. The influence of spatial confinement on the structure and properties of TPs deserves careful study as it is essential for understanding the basic properties of TPs and important for their applications.

In this work we consider theoretically quasi-onedimensional structures formed by narrow and long metal stripes deposited on the BM surfaces [Fig. 1(a)]. Such a stripe is expected to be responsible for the formation of a confinement potential for TPs in a transverse direction, and thus it would be acting as a waveguide. We optimize the geometrical parameters of the stripe which are necessary for efficient TP confinement. The optimized one-dimensional (1D) metallic stripes efficiently confining TP modes would be of immediate use in optical integrated circuits. The dependence of both energy and the loss rate of TP modes on geometric parameters of the stripe is revealed in this work.

\section{THE STRUCTURE UNDER STUDY}

To be specific, we consider here a BM formed by a stack of 40 pairs of GaAs/AlAs $\lambda / 4 n$ layers with a thin silver stripe deposited on the top of the upper semiconductor layer (see Fig. 1). The stripe is assumed to be infinite along the $z$ axis, which is the direction of TP propagation. In the transverse plane it has a rectangular shape with the thickness $h$ and the width $w$. Apart from the stripe all other elements are assumed to be infinite in the $y$ direction. The thicknesses of the GaAs/AlAs layers were taken to correspond to the Bragg quarter-wave condition at the Bragg frequency $\hbar \omega_{B}=1.46 \mathrm{eV}$ $\left(\lambda_{B} \simeq 850 \mathrm{~nm}\right)$. This specific choice of the frequency is dictated by proximity to the exciton resonance in GaAs that would be essential in the context of investigation of $1 \mathrm{D}$ Tamm plasmon polaritons. For simulations we use dispersiveless refractive indexes of GaAs, $n_{\mathrm{GaAs}}=3.7$, and $\mathrm{AlAs}$, $n_{\mathrm{AlAs}}=3.0$. The choice of silver as the material for the stripe is dictated by the fact that it has low absorption in the considered spectral domain. The dielectric permittivity of $\mathrm{Ag}$ is described by the Drude formula, $\varepsilon_{A g}=1-\frac{\omega_{p}^{2}}{\omega(\omega-i \gamma)}$, with material parameters $\hbar \omega_{p}=9.01 \mathrm{eV}$ and $\hbar \gamma=0.018 \mathrm{eV}$ corresponding to the textbook data [20].

The analysis of the structure of Tamm modes propagating along the stripe is performed by means of the finite-element method realized with the commercial software COMSOL. It solves the vectorial wave equation

$$
\vec{\nabla} \times(\vec{\nabla} \times \vec{E})-k_{0}^{2} \varepsilon(x, y) \mu \vec{E}=0
$$

for the electric field $\vec{E}=\vec{E}(x, y) e^{i k_{z} z} e^{i \omega_{\mathrm{tp}} t-\gamma_{\mathrm{pp}} t}$ for the given value of a real wave vector $k_{z}$ considering the complex eigenfrequency $\omega_{\mathrm{tp}}+i \gamma_{\mathrm{tp}}$ as an unknown variable. Here $k_{0}$ is a free-space wave vector, $\varepsilon(x, y)$ is a distribution of the electric permittivity in the plane perpendicular to the propagation direction, and $\mu$ is magnetic permeability, which is taken to be homogeneous. For simulations we suppose that the structure is limited by air on the top, and it is deposited on a GaAs substrate, as shown in Fig. 1(a). For modeling open boundaries in the transverse direction, perfectly matched layers were employed. In order to verify the accuracy of our numerical approach we calculated first the field distribution for the TP mode formed beneath a 50-nm-thick silver film in the case of normal incidence of transverse electric excited light $\left(k_{z}=0\right)$. The numerically calculated absolute value of the electric field amplitude $E_{z}$ is shown by red circles in Fig. 1(b). We check the obtained result with the transfer-matrix method, which provides a rigorous solution for this problem [8] [black curve in Fig. 1(b)]. A reliable coincidence of both results justifies the employed numerical method. 


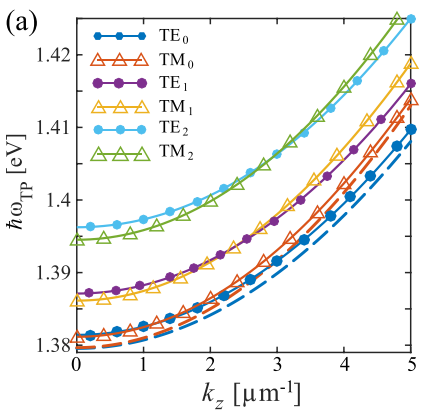

(c)

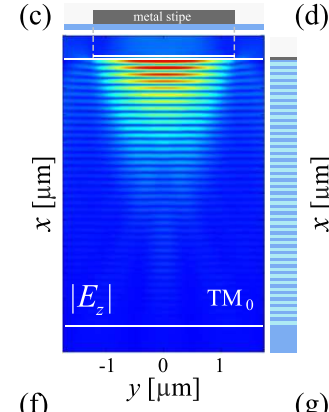

(d)

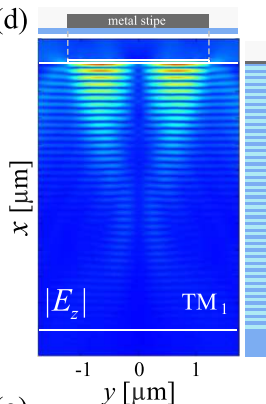

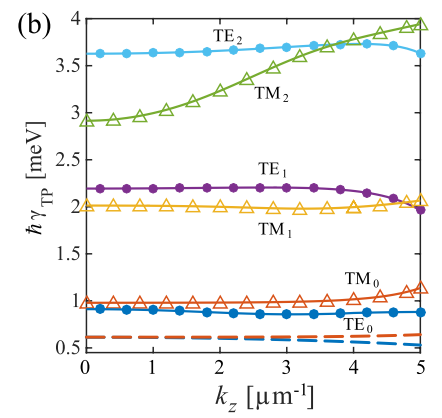

(e)
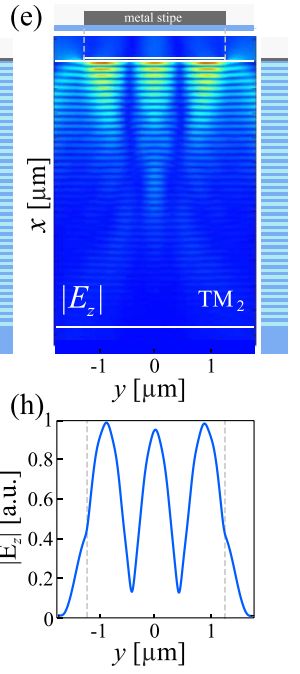

FIG. 2. Properties of modes of a TP waveguide. (a) Dispersion and (b) loss rates for various Tamm modes localized beneath the silver stripe with width $w=2.5 \mu \mathrm{m}$ and thickness $h=50 \mathrm{~nm}$. Solid lines with circles and triangles indicate quasi-TE and quasi-TM Tamm modes, respectively. Blue and red curves correspond to fundamental $\mathrm{TE}_{0}$ and $\mathrm{TM}_{0}$ modes, while excited modes (from bottom to top) are indicated by yellow $\left(\mathrm{TM}_{1}\right)$, purple $\left(\mathrm{TE}_{1}\right)$, green $\left(\mathrm{TM}_{2}\right)$, and cyan $\left(\mathrm{TE}_{2}\right)$ curves. Dashed lines correspond to the case of a continuous-metal layer: TM-polarized plasmons are shown in red, and TE-polarized plasmons are shown in blue. (c)-(e) The distribution of the electric field component $\left|E_{z}\right|$ for three lowest quasi-TM TPs for $k_{z}=4 \mu \mathrm{m}^{-1}$. For each panel the position of the metal layer in a horizontal plane is schematically indicated at the top. The vertical sequence of material layers is illustrated on the right. (f)-(h) Profiles of $\left|E_{z}\right|$ along the $y$ direction in the middle of the top GaAs layer for the modes shown above. Vertical dashed lines indicate the position of the stripe. The red dashed curve in (f) corresponds to the approximated solution (5).

\section{TRANSPORT PROPERTIES OF 1D TAMM PLASMONS: DISPERSION AND PROPAGATION LENGTH}

Observation of confined TPs beneath a metal island reported in $[7,13,18,19]$ makes one expect a similar TP confinement in the waveguide configuration as well. However, in the latter case TPs confined in the transverse direction can propagate along the stripe. Thus, in addition to the distribution of the field the dispersion of TPs, i.e., the dependence of the real part of the eigenfrequency $\omega_{\text {tp }}$ on the propagation constant $k_{z}$, should be investigated.

Our simulations reveal a discrete set of Tamm eigenmodes of the considered stripe waveguide structure (see Fig. 2).
These states arise due to the confinement along the $y$ axis provided by the stripe and thus resemble modes of a slab waveguide [21]. There is a fundamental mode shown in Figs. 2(c) and 2(f) which is labeled by the subscript 0 and a number of excited modes labeled by the subscript $m$, which corresponds to the number of nodes in the field distribution along the $y$ axis [see Figs. 2(d), 2(g) 2(e), and 2(h)]. All these waveguide modes exist in both transverse-magnetic (TM) and transverse-electric (TE) configurations and have parabolic dispersions with slightly different effective masses which are on the order of $3 \times 10^{-5}$ of the free-electron mass.

In contrast to free space where the electric and magnetic field vectors are oriented in the plane perpendicular to the propagation direction, in the structure we consider, the symmetry is broken in the $x-y$ plane, which is why the structure of the electromagnetic field in the eigenmodes is more complex. According to the theory of waveguides [22], the magnetic (electric) field of the TM (TE) modes is perpendicular to the propagation direction, i.e., to the $z$ axis. However, due to the confinement in the $y$ direction the observed Tamm modes are actually quasi-TM and quasi-TE. This means that all six Cartesian components of the electric $\vec{E}$ and magnetic $\vec{H}$ vectors are nonzero for these modes. However, for wide stripes, $w \gg \lambda_{B} / n$, the influence of the discontinuity along the $y$ axis is negligible, and the dominating components are those which are nonzero in purely TE and TM modes forming under the continuous-metal film. They are the $H_{z}, H_{x}$, and $E_{y}$ components for TE modes and $E_{z}, E_{x}$, and $H_{y}$ for TM modes. The corresponding field structures are schematically shown in Fig. 1(c). The structure of the TM mode can be completely described by the longitudinal component of the electric field $E_{z}$ collinear with the propagation direction [22]. This limit should be valid for wide stripes as well. That is why we describe the TM modes by only the $E_{z}$ component [its distribution is shown in Figs. 2(c)-2(h)], while TE Tamm plasmons are described fully by the $H_{z}$ component.

The rate of losses for excited waveguide modes (with an index above zero) are sufficiently higher than for fundamental modes. Although the spectral gap between excited modes of the same polarization grows as the mode number $m$ increases, the loss rate grows faster. That is why only the three lowest modes (for both TE and TM modes) are shown in Fig. 2. The spectral gaps between higher modes are comparable with or smaller than the widths of these modes.

The value of the decay rate of the Tamm plasmon mode is governed by several loss mechanisms. The first one is the radiative emission in the $x$ direction from the sides of both the Bragg mirror and the stripe of finite thickness. The second is the light absorption in the metal layer. Both these mechanisms are characteristic for two-dimensional (2D) Tamm plasmons as well. The finite width of the stripe introduces a supplementary loss channel for 1D Tamm plasmons.

If the width of the stripe is much larger than the wavelength, which corresponds to the condition $w>\lambda_{B}$, the fundamental modes should inherit their properties from the modes formed under a continuous-metal film. Dispersions for the latter are shown by the bottommost dashed curves in Figs. 2(a) and 2(b). The lateral confinement is thus responsible for the blueshift of 


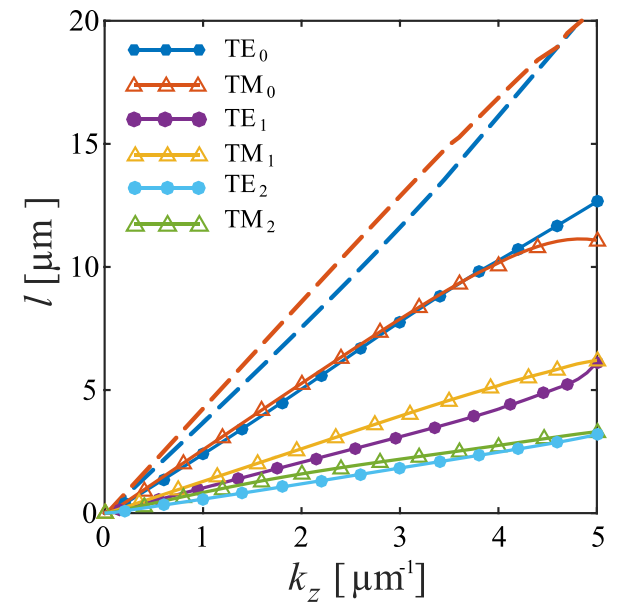

FIG. 3. Propagation lengths of various TP modes. All markers have the same meaning as in Fig. 2

the dispersion curve and for the decrease in the field amplitude from the center of the stripe to its edges [Figs. 2(f)-2(h)]. Since the confinement is due to metal patterning, the presence of open lateral boundaries for TPs leads to additional losses which arise from the leakage of light from under the stripe to the uncovered region of the BM. The contribution of these losses to the overall decay rate of TPs is rather high. Even for fundamental $\mathrm{TM}_{0}$ and $\mathrm{TE}_{0}$ waveguide modes, the decay rate is nearly two times higher than for 2D TPs, and it increases further for excited modes [see Fig. 2(b)].

An important characteristic of waveguide modes is their propagation length $l$. This quantity can be expressed through the group velocity of TPs as $l=v_{g} \gamma_{\mathrm{tp}}^{-1}$, where $v_{g}=\partial \omega_{\mathrm{tp}} / \partial k_{z}$. Taking into account the parabolic dispersion of Tamm modes, one obtains $l=\hbar k_{z} / m^{*} \gamma_{\text {tp }}$. Since the loss rate $\gamma_{\text {tp }}$ demonstrates a weak $k_{z}$ dependence, the value of $l$ grows linearly as the propagation constant increases (see Fig. 3).

Guiding of TPs by the metal stripe causes the reduction of the propagation distance compared to the case of a planar 2D metal layer. For the considered structure only fundamental modes show significant propagation lengths, which are of the order of $10 \mu \mathrm{m}$ for the realistic choice of parameters. So because of the comparatively low group velocities TPs themselves do not have strong advantages in comparison to conventional SPs with respect to the distance of propagation. But the possibility of direct optical excitation of Tamm modes makes them more suitable for applications in optical integrated circuits. Besides, as mentioned before, the Tamm modes can be effectively coupled to exciton polaritons in hybrid Tamm microcavities $[8,10]$. Hybrid Tamm-plasmon polariton (TPP) modes exhibit significantly lower losses than TPs as the major part of the field is concentrated far from the metal in this case. In addition, the energy shift of TPPs is responsible for formation of the local effective potential whose magnitude is dependent on the energy of TPs. The latter is evidently affected by the confinement induced by the stripe. For implementation of TPs in optical circuits, the correlation between geometrical parameters of the metal stripe and the energy of TP modes should be carefully studied.
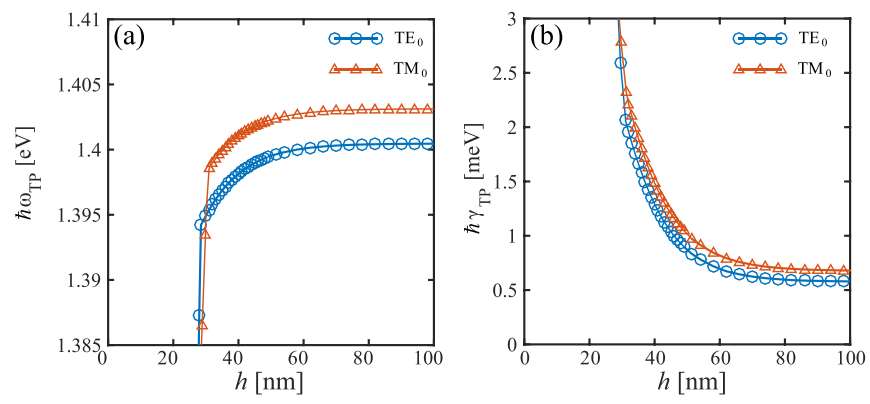

FIG. 4. Dependence of (a) the energy and (b) the loss rate of the fundamental TP modes on the thickness of the stripe for a fixed width $w=2.5 \mu \mathrm{m}$. Propagation constant is $k_{z}=4 \mu \mathrm{m}^{-1}$.

\section{THE EFFECT OF THE STRIPE GEOMETRY ON THE PROPERTIES OF TP MODES}

In this section, keeping the rectangular shape of the stripe, we tune either its width $w$ or thickness $h$. Thinning down of the stripe, as expected, leads to an exponential increase of losses due to leakage of radiation through the metal (see Fig. 4). It is also accompanied by a smooth decrease in the eigenfrequency of TPs by several meV until the mode frequency sharply decreases at $h$ below $30 \mathrm{~nm}$. In this range of thicknesses the losses are rather high, and the propagation lengths are as short as a few units of the wavelength. On the other hand, both the energy and losses saturate for $h \gtrsim 80 \mathrm{~nm}$.

The effect of the stripe width on the energy of TPs is more complex. Figure 5 shows that TE and TM modes behave differently with the variation of $w$. While for the TM-polarized TPs the energy and the decay rate gradually increase with the reduction of the stripe width, for the TE mode these dependencies are modulated. However, the overall trend towards the increase of the mode energy is common for modes of both types. The rate of energy enhancement with the reduction of the width of the stripe is inversely proportional to $w^{2}$, in analogy to the behavior of the quantum confinement energy of an electron in a rectangular potential well with infinite barriers. This analogy is confirmed by the fact that the spectral gaps between different modes (for instance, $\mathrm{TM}_{0}$ and $\mathrm{TM}_{1}$ ) also increase with the reduction of the width $w$.

In the case of tight confinement typical for narrow stripes, the evanescent tails of the field distribution, which spread out of the stripe edges [see Figs. 2(f)-2(h)], become longer. As a result, the coupling to leaky modes of the uncovered BM increases, and the decay rate of TPs grows [see Fig. 5(b)]. It is accompanied by a drastic decrease in the propagation length down to a few micrometers for stripes a micrometer wide. In the opposite limit of wide stripes $(w \gtrsim 4 \mu \mathrm{m}$, not shown in Fig. 5) the decay rate $\gamma_{\text {tp }}$ saturates and approaches its value typical for 2D TPs. Thus the fabrication of metal patterns of a few micrometers is a reasonable trade-off between the demands for low loss rate and miniaturization.

Note that a similar steep increase of coupling to the leaky modes would occur if we fixed the stripe width and increased the wavelength of TPs tuning the Bragg frequency of the BM. However, the losses due to the absorption in the metal layer decrease for longer wavelengths. Thus for wide stripes, where the absorption losses are dominant, the longer wavelengths 

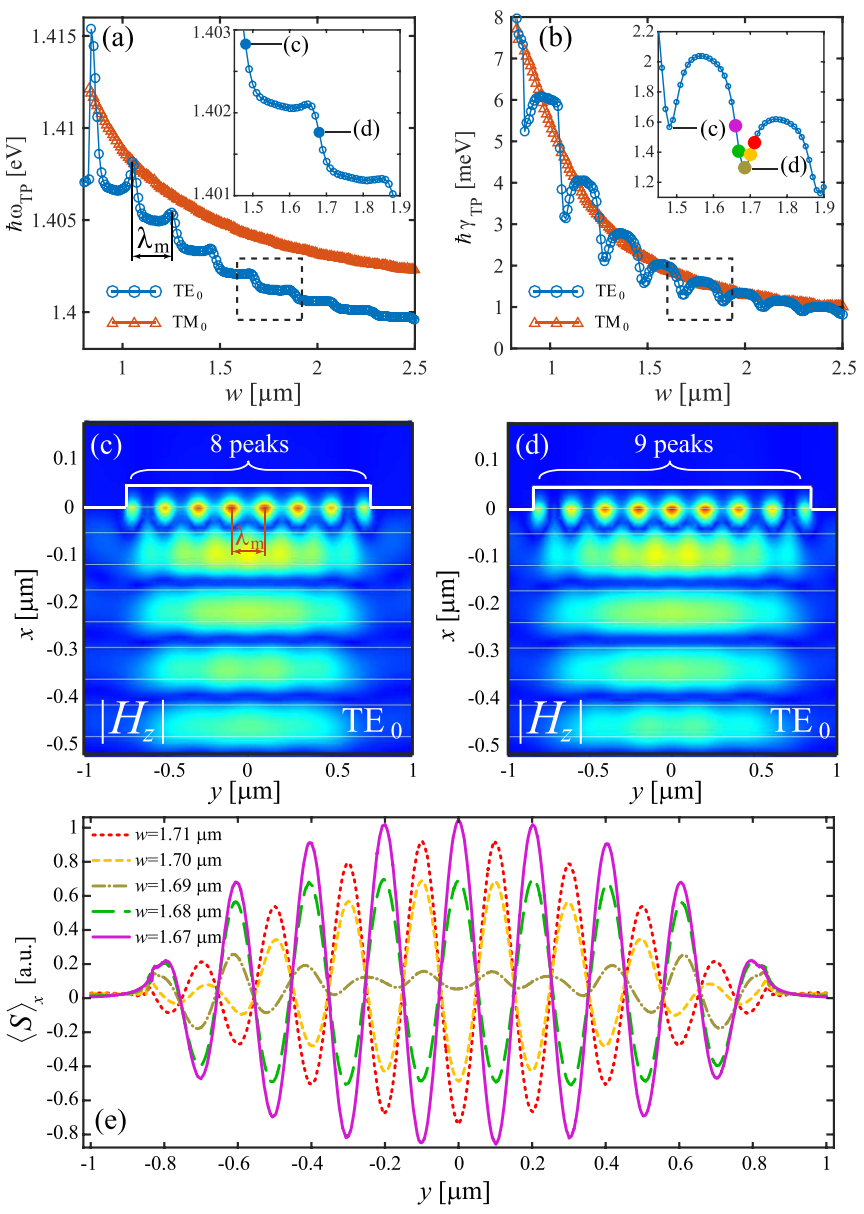

FIG. 5. The dependence of (a) the energy and (b) the loss rate of the fundamental TP modes on the width of the stripe for the fixed thickness $h=50 \mathrm{~nm}$. The wave-vector projection to the propagation direction is $k_{z}=4 \mu \mathrm{m}^{-1}$. The insets in (a) and (b) show magnified images of the areas in dashed frames. (c) and (d) The distribution of the absolute value of the $z$ component of the magnetic field $\left|H_{z}\right|$ for the TE mode for different values of the stripe width: $w=1.49 \mu \mathrm{m}$ in (c) and $w=1.69 \mu \mathrm{m}$ in (d). (e) The time-averaged Poynting vector projection to the vertical direction in the vicinity of the bottom surface of the metallic stripe for various values of the stripe width. The values of the loss rates of the Tamm modes corresponding to these curves are shown in the inset in (b) by the colored circles. The color of the circle corresponds to the color of the curve in (e).

of TPs are beneficial for the maximization of the propagation length. If the stripe width is comparable to the TP wavelength, the leakage of the light from under the stripe dominates other mechanisms of losses, which leads to a drastic reduction of the propagation length.

The nontrivial behavior of TE modes revealed in our numerical simulations deserves special attention. The dependence of the TP energy on the stripe width shown in Fig. 5(a) demonstrates the presence of distinct maxima located nearly equidistantly with respect to the variation of $w$. The same is also valid for the loss rate $\gamma_{\mathrm{tp}}$, but the positions of the maxima are shifted by approximately a quarter of the period [Fig. 5(b)]. It is important to note that the period of modulation $\lambda_{m}$ is smaller than the TP wavelength in either of the semiconductor layers.
Our simulations demonstrate that the stripe-width dependence of the energy of TE-polarized TPs correlates with the field distribution in these modes. Overall, the field profile of the TE mode in the $x y$ plane resembles the field distribution of the TM mode shown in Fig. 2(c). The striking difference is observed in the region close to the metal/semiconductor interface, where the field amplitude is modulated along the $y$ axis, which is across the stripe [see Figs. 5(c) and 5(d)]. Note that the distance between the maxima of the magnetic field component $\left|H_{z}\right|$ exactly matches the period of the modulation $\lambda_{m}$ shown in Figs. 5(a) and 5(b). Those TE modes for which the stripe width differs by $\lambda_{m}$ have similar field structures [see Figs. 5(c) and 5(d)]. The only difference is that the number of field maxima differs by 1 .

The complexity of the field structure in the vicinity of the metal surface is indicative of the admixture of the SP which is excited by the TE-polarized TP in this case. We emphasize that the wave-vector projections of the investigated TPs lie within the light line, $k_{z}<\omega_{\mathrm{tp}} / c$. This means that the excitation of the waveguide SPs propagating in the $z$ direction [23] would be impossible due to the momentum inconsistency. However, that is not the case for the SPs propagating across the stripe. It is well known that SPs can be excited without gratings or prisms in systems that do not conserve the in-plane wave vector of light. In our case the discontinuity of the metal in the $y$ direction enables the excitation of SPs at opposite edges of the stripe that bridge the momentum gap between SPs and TPs.

Excited in this way, SPs propagate across the stripe towards each other and interfere, causing the formation of the interference pattern shown in Fig. 6(a). These patterns should be detectable in the near-field measurements and have much in common with the standing waves of surface plasmon polaritons excited between a pair of thin slits cut out of a metal film which were investigated recently both experimentally and theoretically [24]. The similar field structure was also predicted to describe the plasmon-assisted two-slit Young's experiment [25].

Further evidence for the intermixture of TP and SP modes in the stripe comes from a comparison of the period of modulation of the field magnitude and the wavelength of the corresponding SP. In the case of SPs excited at the boundary between semiinfinite dielectric and metal domains the wavelength is given by [1]

$$
\lambda_{\mathrm{sp}}=\operatorname{Re}\left[\frac{2 \pi \mathrm{c}}{\omega_{\mathrm{sp}}} \sqrt{\frac{\varepsilon_{\mathrm{Ag}}+\varepsilon_{\mathrm{GaAs}}}{\varepsilon_{\mathrm{Ag}} \varepsilon_{\mathrm{GaAs}}}}\right],
$$

where $\varepsilon_{\mathrm{GaAs}}=n_{\mathrm{GaAs}}^{2}$ and $\omega_{\mathrm{sp}}=\omega_{\mathrm{tp}}$. For the considered set of parameters Eq. (2) yields the value $\lambda_{\mathrm{sp}}=197 \mathrm{~nm}$, which matches the modulation period $\lambda_{m}$ extracted from the numerical data shown in Fig. 5. Variation of the TP eigenfrequency $\omega_{\text {tp }}$ by tuning the Bragg frequency of the BM demonstrates good agreement between the results of the numerical simulations and predictions of Eq. (2) over the wide range of frequencies [see Fig. 6(b)]. The residual discrepancy should be attributed to the effect of the finite thickness of the stripe, which shifts the eigenfrequency of the SP mode [23].

Note that the discussed SPs are excited only by the TEpolarized TPs. The reason lies in the polarization of these 

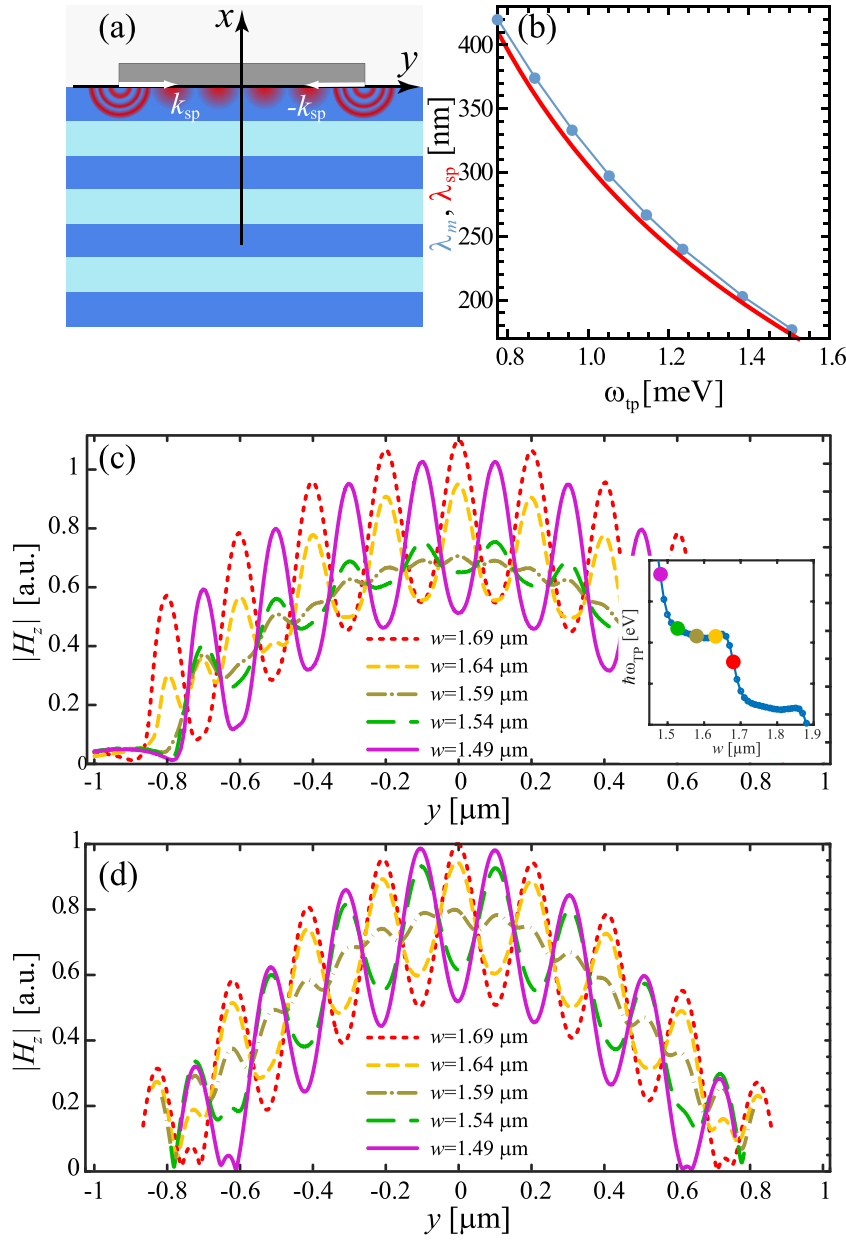

FIG. 6. (a) Schematic representation of the excitation of the SPs at the opposite edges of the metal stripe and the formation of the interference pattern. (b) Blue points correspond to the period of modulation $\lambda_{m}$ of the function $\omega_{\mathrm{tp}}(w)$ for various frequencies of the TE TP mode corresponding to different Bragg frequencies. The red line corresponds to the wavelength of the SP mode $\lambda_{\text {sp }}$ excited at the boundary between the semi-infinite silver and GaAs domains calculated using Eq. (2). (c) and (d) The distribution of the absolute values of the magnetic field component $\left|H_{z}\right|$ along the $y$ direction at the bottom of the metal stripe $(x=0)$. (c) shows the results of numerical simulations, and (d) is calculated using the model equations (3)-(6). For all the panels $h=50 \mathrm{~nm}$.

modes. The SPs can be efficiently excited by a Tamm mode whose polarization coincides with one of the SPs. The wellknown fact that SPs exist only in the TM configuration [1] should not confuse us since in our case the structure of the SP field is actually transverse magnetic with respect to the propagation direction, i.e., the $y$ axis. In the limit of the wide stripes the SP mode propagating along the $y$ axis possesses $E_{x}, E_{y}$, and $H_{z}$ components [1], as schematically shown in Fig. 1(c). It is clearly seen that the polarization of the standing SP mode is close to the polarization of the TE Tamm waveguide mode. On the contrary, its field is almost orthogonal to the pure TM mode [see Fig. 1(c)]. Note that for narrow stripes all the field components become nonvanishing, as mentioned before. In this case, SPs are excited by TM Tamm modes as well. However, their amplitudes are tiny in this case since the dominant field components remain the same as in the case of an infinitely wide stripe.

\section{THE MODEL DESCRIBING THE COUPLING BETWEEN TPS AND SPS}

In order to explain qualitatively the complicated structure of a TE-polarized TP we represent it as a superposition of a confined Tamm mode and SP excited at the metal/dielectric interface. Namely, for the magnetic field component we write

$$
H_{z}=H_{z}^{\mathrm{tp}}+H_{z}^{\mathrm{sp}} .
$$

Here

$$
H_{z}^{\mathrm{tp}}=F(y) T(x) e^{i k_{z} z}
$$

is a pure TP mode, confined beneath the stripe. $T(x)$ is a Tamm mode uniform along the $y$ axis formed under the continuousmetal film. The prefactor $F(y)$ accounts for the decay of the field from the center of the stripe to its sides. It should resemble the profile of the TM mode shown in Figs. 2(f)-2(h). Following the analogy between the considered system and the problem of a particle in a potential box, we assume for the fundamental $\mathrm{TE}_{0}$ mode

$$
F(y)=\left\{\begin{array}{ccc}
h_{\mathrm{tp}} \cos \left(\frac{\pi y}{w}\right), & \text { for } & |y| \leqslant w / 2 \\
0, & \text { for } & |y|>w / 2
\end{array}\right.
$$

where $h_{\mathrm{tp}}$ is a complex amplitude of the TP mode. Here for the sake of simplicity we assume that the stripe represents an effective potential well with an infinite depth for the TPs. The profile (5) is shown by the dashed red line in Fig. 2(f), while the blue curve corresponds to the actual field profile calculated numerically. Although the approximation used is inaccurate for regions close to the stripe edges, it fits well the field profile at the central part of the structure and thus seems to be a good approximation for our qualitative analysis.

The term $H_{z}^{\text {sp }}$ in Eq. (3) describes the structure of the SP field localized at the metal/dielectric interface. As mentioned before, the bottom edges of the stripe scatter the field into the plasmonic channel, acting as point sources for the SP field. Thus for the field inside BM we write

$$
\begin{aligned}
H_{z}^{\mathrm{sp}}= & h_{\mathrm{sp}}\left(e^{-i k_{\mathrm{sp}}(y-w / 2)} e^{-\frac{(y-w / 2)}{2 L_{\mathrm{sp}}}}\right. \\
& \left.+e^{i k_{\mathrm{sp}}(y+w / 2)} e^{-\frac{(y+w / 2)}{2 L_{\mathrm{sp}}}}\right) e^{\beta x} e^{i k_{z} z},
\end{aligned}
$$

where $k_{\mathrm{sp}}=2 \pi / \lambda_{\mathrm{sp}}, h_{\mathrm{sp}}$ is a complex mode amplitude, and $\beta$ is an attenuation constant which characterizes the damping of the SP field in the $x$ direction. Damping exponents in Eq. (6) are responsible for the decay of the amplitude along the $y$ direction characterized by the propagation length of the SP $L_{\mathrm{sp}}=\left(2 \operatorname{Im}\left[k_{\mathrm{sp}}\right]\right)^{-1}$, which is about $5 \mu \mathrm{m}$ for the considered parameters. This value is longer than the typical width of the stripe considered here, and we can safely omit the corresponding decay factors from Eq. (6) without the loss of generality.

The numerically calculated structure of the magnetic field component $\left|H_{z}\right|$ at the bottom surface of the stripe is shown in Fig. 6(c). The results corresponding to various values of $w$ are presented for comparison. Dotted red and solid magenta curves characterized by the highest peak-to-valley ratio correspond to the modes shown in Figs. 5(c) and 5(d). 
These modes are excited at the stripe widths which differ by $\lambda_{m}$. The states excited at the intermediate values of $w$ exhibit a less pronounced modulation of the field amplitude. With the reduction of the stripe width the peak-to-valley ratio gradually decreases, almost reaching a smooth field profile at $w=1.59 \mu \mathrm{m}$. Then the modulation contrast recovers, but the number of peaks is reduced by 1 .

Such behavior can be interpreted as follows. The variation of the stripe width changes the phase difference between the SP waves and thus affects the interference pattern. So the maximum peak-to-valley ratio is obtained where the width $w$ is a multiple of the SP wavelength disregarding the phase shift between the amplitudes of the SP and TP fields, $\Delta \varphi=$ $\arg \left(h_{\mathrm{tp}}\right)-\arg \left(h_{\mathrm{sp}}\right)$. The strongest modulation condition can be straightforwardly derived from Eqs. (3)-(6):

$$
w=(p+\Delta \varphi / \pi) \lambda_{\mathrm{sp}},
$$

where $p$ is an integer number. For instance, for the mode shown in Fig. 5(c) $p=7$, and $p=8$ for Fig. 5(d).

The field profile obtained from the analytical model (3)-(6) is shown in Fig. 6(d). In this particular case the best agreement between the approximated model and the numerical calculations is obtained for $\Delta \varphi=\pi / 3,\left|h_{\mathrm{tp}}\right|=0.75$, and $\left|h_{\mathrm{sp}}\right|=0.25$, where the maximum of the field $H_{z}$ was normalized to unity.

Note that for those values of the stripe width for which the constructive interference of SPs occurs the losses of the TP mode are minimal [see the inset in Fig. 5(b)]. This seems contradictory since the appearance of SPs is usually associated with the increase in losses due to the higher penetration of the field into the metal layer. The latter is actually true in our case. For the infinitely wide stripe (when there is no admixture of SPs into the TE Tamm mode) the loss rate for the latter is lower than for the TM mode [see Figs. 2(b) and 5(b)]. At the same time for stripes with finite widths the SP affects the loss rate of the TE mode. So for some values of $w$ the TE mode is even more dissipative than the TM mode.

In order to estimate the impact of the admixture of the SP on the loss rate of the TE-polarized TP we evaluate the average energy flux transmitted through the metal stripe that is given by the real part of the $x$ component of the time-averaged complex Poynting vector $\vec{S}$ defined as

$$
\left\langle S_{x}\right\rangle=\frac{1}{2} \operatorname{Re}\left[E_{y} H_{z}^{*}-E_{z} H_{y}^{*}\right]
$$

The results computed from the spatial distribution of the mode field obtained with numerical simulations are plotted in Fig. 5(e). Different curves correspond to the values of the stripe width belonging to the narrow range around the bottom of a single local minimum of $\gamma_{\mathrm{tp}}(w)$ dependence shown in Fig. 5(b). Note that the dash-dotted brown curve for which the amplitude of the energy flux through the stripe is minimal corresponds to the case of the constructive interference of SPs [red curves in Figs. 6(c) and 6(d)]. Since the energy flux through the stripe is the lowest in this particular case the Ohmic losses are also minimized.

The behavior of the energy flux $\left\langle S_{x}\right\rangle$ can be described with the model (3)-(6). The standard expression for the electric field component $E_{y}$ for TE mode is derived [22] from the wave equation (1):

$$
E_{y}=-\frac{i \mu \omega_{\mathrm{tp}}}{k_{0}^{2} \varepsilon-k_{z}^{2}} \frac{\partial H_{z}}{\partial x} .
$$

We disregard the $E_{z}$ and $H_{y}$ components in Eq. (8) as they are negligible both for the TE Tamm mode and for SP [see Fig. 1(c)]. Then substituting Eqs. (3)-(6) and (9) in Eq. (8), we obtain

$$
\begin{aligned}
\left\langle S_{x}\right\rangle= & \frac{\mu \omega_{\mathrm{tp}}}{k_{0}^{2} \varepsilon-k_{z}^{2}}\left|h_{\mathrm{tp}}\right|\left|h_{\mathrm{sp}}\right| \cos \left(\frac{\pi y}{w}\right) \cos \left(k_{\mathrm{sp}} y\right) e^{\beta x} \\
& \times\left(\beta T-\frac{\partial T}{\partial x}\right) \sin \left(k_{\mathrm{sp}} w / 2-\Delta \varphi\right) .
\end{aligned}
$$

The energy flow vanishes if the last multiplier in (10) is zero. Hence we obtain $w=(p+\Delta \varphi / \pi) \lambda_{\text {sp }}$, which coincides with the criterion (7) for the stripe width for which the constructive interference of the SP waves occurs.

\section{CONCLUSION}

Patterning of the metal layer deposited on the surface of the BM offers a powerful tool for confining the TP mode as well as for controlling its structure and energy. Localization of light under the metal stripe deposited at the BM leads to the formation of a discrete set of waveguide modes which lie within the light line and thus can be directly excited from the outside. The analysis shows that the best confinement properties are obtained for fundamental modes where the width of the structure is about a few micrometers and its thickness is of the order of tens of nanometers. The finite width of the stripe leads to the excitation of localized surface plasmon modes which are efficiently coupled to only the TE-polarized TPs. The presence of SPs affects both the confinement energy and the loss rates of the TE Tamm modes which demonstrate periodic dependencies on the stripe width. The discussed dependence of the frequency of Tamm mode on the geometrical parameters of the stripe allows for the engineering of nontrivial potential landscapes along the stripe which can be done by the modulation of either its width or thickness.

The investigated properties of 1D TPs can be easily generalized to a more complex case of two-dimensional TP networks. The discreet set of confined modes, the field profile, and the effect of excitation of SP interference patterns should also be typical for 2D localized TPs. This analysis paves the way for the realization of integrated optical networks based on Tamm-plasmon polaritons.

\section{ACKNOWLEDGMENTS}

I. Yu.Ch. acknowledges the support from RFBR Grant No. 16-32-60102 and partial support from the President of the Russian Federation for state support of young Russian scientists, Grant No. MK-2988.2017.2. E.S.S. acknowledges the support from RFBR Grant No. 16-32-60104 and the partial support from the President of the Russian Federation for state support of young Russian scientists, Grant No. MK-8031.2016.2. S.V.K. acknowledges the support from RFBR Grant No. 1632-60067-mol_a_dk and partially support from the President of the Russian Federation for state support of young Russian scientists, Grant No. MK-2842.2017.2. S.M.A. acknowledges 
the support from the Ministry of Education and Science of the Russian Federation, Project No. 16.1123.2017/4.6, and from RFBR Grant No. 15-59-30406. A.V.K. acknowledges Saint-Petersburg State University for a research grant (Grant
No. 11.34.2.2012), the support from the EPSRC Programme grant on Hybrid Polaritonics (Grant No. EP/M025330/1), and the partial support from the 551 EU HORIZON 2020 RISE project CoExAn (Grant No. 644076).
[1] S. A. Maier, Plasmonics: Fundamentals and Applications (Springer, Berlin, 2007).

[2] M. Kaliteevski, I. Iorsh, S. Brand, R. A. Abram, J. M. Chamberlain, A. V. Kavokin, and I. A. Shelykh, Phys. Rev. B 76, 165415 (2007).

[3] I. Tamm, Zh. Eksp. Teor. Fiz. 3, 34 (1933).

[4] M. E. Sasin, R. P. Seisyan, M. A. Kaliteevski, S. Brand, R. A. Abram, J. M. Chamberlain, A. Y. Egorov, A. P. Vasil'ev, V. S. Mikhrin, and A. V. Kavokin, Appl. Phys. Lett. 92, 251112 (2008).

[5] H. Liu, X. Sun, F. Yao, Y. Pei, H. Yuan, and H. Zhao, Plasmonics 7, 749 (2012).

[6] B. I. Afinogenov, V. O. Bessonov, A. A. Nikulin, and A. A. Fedyanin, Appl. Phys. Lett. 103, 061112 (2013).

[7] S. Azzini, G. Lheureux, C. Symonds, J.-M. Benoit, P. Senellart, A. Lemaitre, J.-J. Greffet, C. Blanchard, C. Sauvan, and J. Bellessa, ACS Photonics 3, 1776 (2016).

[8] M. Kaliteevski, S. Brand, R. A. Abram, I. Iorsh, A. V. Kavokin, and I. A. Shelykh, Appl. Phys. Lett. 95, 251108 (2009).

[9] S. K. S.-U. Rahman, T. Klein, S. Klembt, J. Gutowski, D. Hommel, and K. Sebald, Sci. Rep. 6, 34392 (2016).

[10] C. Symonds, A. Lemaitre, E. Homeyer, J. Plenet, and J. Bellessa, Appl. Phys. Lett. 95, 151114 (2009).

[11] N. Lundt, S. Klembt, E. Cherotchenko, S. Betzold, O. Iff, A. V. Nalitov, M. Klaas, C. P. Dietrich, A. V. Kavokin, S. Höfling et al., Nat. Commun. 7, 13328 (2016).

[12] T. Hu, Y. Wang, L. Wu, L. Zhang, Y. Shan, J. Lu, J. Wang, S. Luo, Z. Zhang, L. Liao et al., Appl. Phys. Lett. 110, 051101 (2017).
[13] O. Gazzano, S. M. de Vasconcellos, K. Gauthron, C. Symonds, J. Bloch, P. Voisin, J. Bellessa, A. Lemaitre, and P. Senellart, Phys. Rev. Lett. 107, 247402 (2011).

[14] S. Núñez-Sánchez, M. Lopez-Garcia, M. M. Murshidy, A. G. Abdel-Hady, M. Serry, A. M. Adawi, J. G. Rarity, R. Oulton, and W. L. Barnes, ACS Photonics 3, 743 (2016).

[15] O. Gazzano, S. Michaelis de Vasconcellos, K. Gauthron, C. Symonds, P. Voisin, J. Bellessa, A. Lemaître, and P. Senellart, Appl. Phys. Lett. 100, 232111 (2012).

[16] C. E. Little, R. Anufriev, I. Iorsh, M. A. Kaliteevski, R. A Abram, and S. Brand, Phys. Rev. B 86, 235425 (2012).

[17] H. Liu, J. Gao, Z. Liu, X. Wang, H. Yang, and H. Chen, J. Opt. Soc. Am. B 32, 2061 (2015).

[18] C. Symonds, G. Lheureux, J.-P. Hugonin, J.-J. Greffet, J. Laverdant, G. Brucoli, A. Lemaître, P. Senellart, and J. Bellessa, Nano Lett. 13, 3179 (2013).

[19] G. Lheureux, S. Azzini, C. Symonds, P. Senellart, A. Lemaître, C. Sauvan, J.-P. Hugonin, J.-J. Greffet, and J. Bellessa, ACS Photonics 2, 842 (2015).

[20] P. B. Johnson and R.-W. Christy, Phys. Rev. B 6, 4370 (1972).

[21] R. Harrington, Time-Harmonic Electromagnetic Fields (Wiley, New York, 1961).

[22] D. Marcuse, Light Transmission Optics (Van Nostrand Reinhold, New York, 1972).

[23] P. Berini, Phys. Rev. B 63, 125417 (2001).

[24] P. Dvořák, T. Neuman, L. Břínek, T. Šamořil, R. Kalousek, P. Dub, P. Varga, and T. Šikola, Nano Lett. 13, 2558 (2013).

[25] H. F. Schouten, N. Kuzmin, G. Dubois, T. D. Visser, G. Gbur, P. F. A. Alkemade, H. Blok, G. W. 't Hooft, D. Lenstra, and E. R. Eliel, Phys. Rev. Lett. 94, 053901 (2005). 